Received: 01.03.2021

Revised: 02.04.2021

Accepted: 23.04 .2021

DOI: $10.17804 / 2410-9908.2021 .2 .022-029$

\title{
GRAVITATIONAL PRINCIPLE OF MINIMUM PRESSURE FOR INCOMPRESSIBLE FLOWS
}

\author{
E. Yu. Prosviryakov \\ Institute of Engineering Science, Ural Branch of the Russian Academy of Sciences, \\ 34 Komsomolskaya St., Ekaterinburg, 620049, Russian Federation \\ https://orcid.org/0000-0002-2349-7801 《evgen_pros@mail.ru \\ Corresponding author. E-mail: evgen_pros@mail.ru \\ Address for correspondence: 34 Komsomolskaya St., Ekaterinburg, 620049, Russian Federation \\ Tel.: +7 (343) 37535 76; fax: +7 (343) 3745330
}

We consider the flows of ideal (Euler equations) and Newtonian viscous (Navier-Stokes equations) incompressible fluids in the gravitational field of mass forces. In this case, the gravitational field created by the liquid itself (self-gravity) is also taken into account. It is shown that some well-known principles of maximum pressure, according to which either the pressure is constant in the flow region, or the minimum pressure is reached at the boundary of this region if the forces of self-gravity are taken into account, exclude the case of constant pressure. It is also demonstrated that self-gravity makes it impossible for waves and solitons to pass with pressure minima to the surface of a body flown around by a viscous fluid.

Keywords: principle of minimum pressure, vortex flows, ideal incompressible fluid, viscous incompressible fluid.

\section{References}

1. $\quad$ Landau L.D., Lifshitz E.M. Fluid Mechanics (Volume 6 of A Course of Theoretical Physics) [Original Russian text published in Landau L.D., Lifshitz E.M. Teoreticheskaya fizika. T. 6. Gidrodinamika. Moskva, FIZMATLIT Publ., 1986, 736 p.], Oxford, Pergamon Press, 1987, 536 p.

2. Privalova V.V., Prosviryakov E.Yu. Vortex flows of a viscous incompressible fluid at constant vertical velocity under perfect slip conditions. Diagnostics, Resource and Mechanics of materials and structures, 2019, iss. 2, pp. 57-70. DOI: 10.17804/2410-9908.2019.2.057-070. Available at: https://dream-journal.org/DREAM_Issue_2_2019_Privalova_V.V._et_al._057_070.pdf

3. Prosviryakov E.Yu. Dynamic equilibria of a nonisothermal fluid. Vestn. Samar. Gos. Tekhn. Univ., Ser. Fiz.-Mat. Nauki [J. Samara State Tech. Univ., Ser. Phys. Math. Sci.], 2018, vol. 22, no. 4, pp. 735-749. DOI: 10.14498/vsgtu1651. (In Russian).

4. Prosviryakov E.Y. Exact Solutions for three-dimensional potential and vorticity Couette flows of an incompressible viscous fluid. Vestnik Natsional'nogo issledovatel'skogo yadernogo universiteta "MIFI", 2015, vol. 4, no. 6, pp. 501-506. DOI: 10.1134/S2304487X15060127. (In Russian).

5. Burmasheva N.V., Prosviryakov E.Y. Thermocapillary Convection of a Vertical Swirling Liquid. Theoretical Foundations of Chemical Engineering, 2020, vol. 54, pp. 230-239. DOI: $10.1134 / \mathrm{S} 0040579519060034$.

6. Prosviryakov E.Y. New class of exact solutions of Navier-Stokes equations with exponen_tial dependence of velocity on two spatial coordinates. Theoretical Foundations of Chemical Engineering, 2019, vol. 53, No. 1, pp. 107-114. DOI: 10.1134/S0040579518060088. 
7. Aristov S.N., Knyazev D.V., Polyanin A.D. Exact solutions of the Navier-Stokes Equations with the linear dependence of velocity components on two space variables. Theoretical Foundations of Chemical Engineering, 2009, vol. 43, no. 5, pp. 642-662. DOI: 10.1134/S0040579509050066.

8. Knyazev D.V. Solving the motion equations of a viscous fluid with a nonlinear dependence between a velocity vector and some spatial variables. Journal of Applied Mechanics and Technical Physics, 2018, vol. 59, pp. 928-933. DOI: 10.1134/S0021894418050218.

9. Hamel G. Ein allgemeiner Satz über den Druck bei der Bewegung volumbeständiger Flüssigkeiten. Monatsh.f. Mathematik und Physik, 1936, vol. 43, pp. 345-363. DOI: 10.1007/ /BF01707614.

10. Batchelor G.K. An Introduction to Fluid Dynamics, University Press, Cambridge, 1970. DOI: $10.1017 / \mathrm{CBO} 9780511800955$.

11. Truesdell C. A First Course in Rational Continuum Mechanics, New York, Academic Press, $1977,295 \mathrm{p}$.

12. Serrin J. Mathematical principles of classical fluid mechanics. In: Fluid Dynamics I/Strömungsmechanik I, ed. C. Truesdell, Encyclopedia of Physics/Handbuch der Physik, vol. 3/8/1, Springer, 1959, pp. 125-263.

13. Sedov L.I. Mechanics of Continuous Media, World Sci., River Edge, NJ, 1997.

14. Sizykh G. B. A sign of the presence of a deceleration point in a plane irrotational perfect gas flow. Trudy MFTI, 2015, vol. 7 (2), pp. 108-112. (In Russian).

15. Burmistrov A.N., Kovalev V.P., Sizykh G.B. Maximum Principle for the Solution of an Elliptic Equation with Unbounded Coefficients. In: Trudy MFTI (Proceedings of MIPT), 2014, vol. 6, no. 4, pp. 97-102. (In Russian).

16. Golubkin V.N., Sizykh G.B. Maximum principle for the Bernoulli function. TsAGI Science Journal, 2015, vol. 46, No. 5, pp. 485-490. DOI: 10.1615/TsAGISciJ.v46.i5.50.

17. Golubkin V.N., Sizykh G.B. Maximum principles in hydrodynamics. In: Proceedings of XI All-Russian Congress on Fundamental Problems of Theoretical and Applied Mechanics, Kazan, August 20-24, 2015. Kazan, Izdatelstvo Kazanskogo (Privolzhskogo) Federalnogo Universiteta Publ., 2015, pp. 989-991. (In Russian).

18. Besportochnyy A.I., Burmistrov A.N., Sizykh G.B. Version of the Hopf Theorem. Trudy MFTI [Proceedings of MIPT], 2016, vol. 8, no. 1, pp. 115-122. (In Russian).

19. Golubkin V.N., Kovalev V.P., Sizykh G.B. Maximum principle for pressure in ideal incompressible fluid flows. TsAGI Science Journal, 2016, vol. 47, iss. 6, pp. 599-609. DOI: 10.1615/ /TsAGISciJ.2017019567.

20. Sizykh G.B. A velocity minimum in a potential fluid flow. Fluid Dynamics, 2017, vol. 52 (3), pp. 345-350. DOI: 10.1134/S0015462817030024.

21. Polyachenko V. L., Fridman A. M., Physics of Gravitating Systems I: Equilibrium and Stability, Rus. transl. [Ravnovesie i ustoycivost` gravitiruyushchikh sistem, Moskva, Nauka Publ., 1976], Springer-Verlag Berlin Heidelberg, 1984.

22. Golubkin V.N., Korolev G.L., Sizykh G.B. Differential properties of pressure in a viscous fluid. TsAGI Science Journal, 2016, vol. 47, iss. 1, pp. 41-49. DOI: 10.1615/TsAGISciJ.2016017060. 
Подана в журнал: 01.03.2021

УДК 517.598

DOI: $10.17804 / 2410-9908.2021 .2 .022-029$

\title{
ГРАВИТАЦИОННЫЙ ПРИНЦИП МИНИМУМА ДАВЛЕНИЯ В ТЕЧЕНИЯХ НЕСЖИМАЕМОЙ ЖИДКОСТИ
}

\author{
Е. Ю. Просвиряков \\ Институт машиноведения УрО РАН, ул. Комсомольская, \\ 34, г. Екатеринбург, Российская Федераџия \\ https://orcid.org/0000-0002-2349-7801 @ evgen_pros@mail.ru \\ Ответственный автор. Электронная почта: evgen_pros@mail.ru \\ Адрес для переписки: ул. Комсомольская, 34, г. Екатеринбург, Российская Федерация \\ Тел.: +7 (343) 375-35-76; факс: 374-53-30
}

Рассмотрены течения идеальной (уравнения Эйлера) и ньютоновской вязкой (уравнения Навье-Стокса) несжимаемых жидкостей в гравитационном поле массовых сил. С учетом гравитационного поля, создаваемого самой жидкостью (самогравитация). Очевидно, что некоторые известные принципы максимума давления, согласно которым или давление постоянно в области течения, или минимум давления достигается на границе этой области при учете сил самогравитации, исключают случай постоянного давления. А самогравитация приводит к невозможности прохождения волн и солитонов с минимумами давления до поверхности тела, обтекаемого вязкой жидкостью.

Ключевые слова: принцип минимума давления, вихревые течения, идеальная несжимаемая жидкость, вязкая несжимаемая жидкость.

\section{1. Введение}

Учет «пренебрежимо малого», но все-таки ненулевого слагаемого в уравнениях Навье-Стокса может приводить к выводам, которые нельзя получить при нулевом значении этого слагаемого. Самым ярким примером этого утверждения в гидродинамике являются ползущие течения - движения вязкой несжимаемой жидкости, характеризующейся очень малым значением числа Рейнольдса [1]. Малое число Рейнольдса указывает на очень маленькую скорость течения или мелкий характерный геометрический масштаб течения, на большое значение молекулярной вязкости жидкости [1].

Важность учета конвективной производной уравнений Навье-Стокса продемонстрирована на примере модификаций течений Куэтта, Пуазейля и их конвективных аналогов в статьях [2-5]. Исследование этих точных решений показывает важность качественных и количественных свойств уравнений Навье-Стокса [2-5]. Несмотря на то, что построение классов точных решений является важной и актуальной задачей гидродинамики, необходимо также развивать другие идеи анализа уравнений движения вязкой несжимаемой жидкости [1, 6-13].

В работе исследован вопрос о месте точек минимума давления. Исследованию этого вопроса уделяли внимание основатели современной гидродинамики [9-13] и посвящают свои работы современные авторы [14-20]. Дело в том, что место точек минимума давления важно для исследования процесса возникновения кавитации [17]. Учет «пренебрежимо малого» слагаемого - силы самогравитации, позволил получить новые утверждения о месте расположения точек минимума давления. 


\section{2. Основные обозначения и уравнения движения}

Рассмотрим течение ньютоновской вязкой несжимаемой жидкости в гравитационном поле. Обозначим через $\mathbf{V}-$ скорость жидкости; $\boldsymbol{\Omega}=\nabla \times \mathbf{V}-$ вектор завихренности; $p$ - давление; $\rho$ - плотность; $\mu$ - динамический коэффициент вязкости; $\varphi$ - потенциал гравитационных сил; $\nabla$ - оператор Гамильтона (набла-оператор). Движение жидкости описано уравнениями Навье-Стокса например в форме Громеки-Ламба [1]:

$$
\begin{gathered}
\frac{\partial \mathbf{V}}{\partial t}+\boldsymbol{\Omega} \times \mathbf{V}=\frac{\mu}{\rho} \Delta \mathbf{V}-\nabla\left(\frac{p}{\rho}+\frac{\mathbf{V}^{2}}{2}+\varphi\right) ; \\
\nabla \cdot \mathbf{V}=0 .
\end{gathered}
$$

Потенциал гравитационных сил $\varphi$ зависит от пространственных координат и от времени [21]. Гравитационная сила, отнесенная к плотности, равна $-\nabla \varphi$. Пусть $A-$ внутренняя точка течения, а $U_{A}$ - некоторая шаровая окрестность точки $A$, целиком расположенная внутри течения. Уменьшая размер рассматриваемого шара $U_{A}$, всегда можно добиться того, что вклад в гравитационную силу $-\nabla \varphi$ в точке $A$, который вносит сама жидкость, находящаяся внутри шара $U_{A}$, можно считать пренебрежимо малым по сравнению со вкладом от оставшейся части жидкости и по сравнению со вкладами, привносимыми Землей, Луной, Солнцем и другими небесными телами. Однако есть одно свойство гравитационного поля в точке $A$, которое, наоборот, не зависит от тел, расположенных вне шара $U_{A}$ (в настоящей работе рассматриваются внешние тела и жидкость, движущиеся с нерелятивистскими скоростями), а зависит только от жидкости, расположенной внутри сколь угодно малого (но не вырожденного в точку) шара $U_{A}$. Это - лапласиан потенциала гравитационных сил. Он зависит только от плотности жидкости в рассматриваемой точке [21]:

$$
\Delta \varphi=4 \pi \rho G,
$$

где $\rho$ - плотность жидкости; $G \approx 6,674 \cdot 10^{-11} \cdot \mathrm{m}^{3} \cdot \mathrm{kg}^{-1} \cdot \mathrm{s}^{-2}-$ универсальная гравитационная постоянная. В отличие, например, от интенсивности магнитного поля при заданной плотности жидкости ни в каком эксперименте эту величину невозможно сделать сколь угодно малой. Ее нельзя изменить каким-либо расположением масс вне рассматриваемой точки течения.

Находя дивергенцию обеих частей уравнения (2.1), с учетом уравнения неразрывности (2.2), исключим слагаемые, отвечающие за нестационарность и вязкость, и получим:

$$
\nabla \cdot(\boldsymbol{\Omega} \times \mathbf{V})=-\Delta\left(\frac{p}{\rho}+\frac{\mathbf{V}^{2}}{2}+\varphi\right)
$$

Повторяя, например, преобразования, выполненные в работе [22] (в этой работе величина $\Delta \varphi$ считалась пренебрежимо малой, и полагалось, что $\Delta \varphi=0)$, приведем уравнение (2.3) к виду: 


$$
\Delta\left[\frac{p}{\rho}\right]=\Omega^{2}-\left(\nabla V_{x}\right)^{2}-\left(\nabla V_{y}\right)^{2}-\left(\nabla V_{z}\right)^{2}-\Delta \varphi,
$$

где $V_{x}, V_{y}$ и $V_{z}$ - компоненты скорости $\mathbf{V}$ в прямоугольной декартовой системе координат Oxyz, $\Omega=|\boldsymbol{\Omega}|$.

Заметим, что уравнение (2.4) верно и для стационарных, и для нестационарных течений, как для вязкой, так и для идеальной жидкости. Это уравнение известно давно. Его использовал Г. Гамель [9] для оценки верхней границы величины завихренности, при которой силы гравитации, созданные самой жидкостью внутри объема со свободными границами, не дают распадаться этому объему на части. Заметим также, что равносильность уравнений (2.3) и (2.4) существенным образом опирается на уравнение (2.2), т. е. на несжимаемость жидкости.

Из теории гармонических функций следует, что если правая часть (2.4) в некоторой области $D$ не больше нуля, то давление $p$, если оно непостоянно в $D$, принимает минимальное значение только на границе области $D$. Именно это свойство использовал Г. Гамель в работе [9].

Из уравнения (2.4) следует, что в некоторых случаях силы самогравитации (т. е. силы гравитации, созданные самой жидкостью) могут оказывать влияние на качественную картину течения (расположение точек минимума давления). Речь идет о тех случаях, когда учет величины $\Delta \varphi$ влияет на знак правой части (2.4). Таким случаям посвящены следующие разделы.

\section{3. Течение с малой завихренностью}

Гамель, в работе [9] показал, что если величина завихренности $\Omega$ не превосходит $\sqrt{8 \pi \rho G}$, то правая часть (2.4) будет отрицательна. Несложно привести пример реального течения с такой малой завихренностью. Плотность жидкой части ядра Земли по разным оценкам составляет около $10^{4} \mathrm{~kg} / \mathrm{m}^{3}$. Соответствующее значение $\sqrt{8 \pi \rho G}$ равно примерно $4,1 \cdot 10^{-3} \mathrm{~s}^{-1}$, что почти в тридцать раз превышает завихренность твердого тела, вращающегося с угловой скоростью Земли. Поэтому можно утверждать, что в жидкой части ядра Земли минимум давления достигается на границе этой жидкой части. Это следствие из результата Г. Гамеля [9] представляется, по-видимому, впервые.

\section{4. Гидростатический принцип минимума давления}

Если жидкость покоится, то уравнение (2.4) принимает вид:

$$
\Delta\left[\frac{p}{\rho}\right]=-4 \pi \rho G
$$

Поэтому в покоящейся несжимаемой жидкости при отсутствии внешних сил, кроме, может быть, гравитационных сил, давление непостоянно по пространству в любой внутренней подобласти и достигает своего минимального значения на границе и только на границе рассматриваемой подобласти. Отсюда, в частности, следует один из результатов данной работы. Он состоит в том, что постоянства давления внутри области невозможно добиться никаким расположением масс снаружи этой области (в гидростатике). 


\section{5. Безвихревое течение}

В общем случае, если можно априори (до решения задачи) указать область течения, в которой правая часть (2.4) отрицательна, то можно априори утверждать, что давление (если оно непостоянно) достигает минимума только на границе этой области. Так, например, это будет верно для безвихревого течения идеальной жидкости, поскольку для идеальной жидкости также верно уравнение (2.3) и, следовательно, верно уравнение (2.4). Для идеальной несжимаемой жидкости можно априори утверждать, что завихренность будет всюду равна нулю, если она равна нулю в набегающем потоке. Отсюда следует, что точки минимума давления (точки зарождения кавитации) в таких течениях расположены на границах областей с гладкими параметрами течения. Это отличается от известного принципа минимума давления в безвихревых течениях идеальной жидкости (в котором не учитывалась сила самогравитации) [10] тем, что в [10] допускался случай всюду постоянного давления.

\section{6. Вязкое обтекание гладкой поверхности}

Правая часть (2.4), как и полностью это уравнение, не содержит вязкие члены. Однако учет вязкости позволяет оценить величину

$$
\Omega^{2}-\left(\nabla V_{x}\right)^{2}-\left(\nabla V_{y}\right)^{2}-\left(\nabla V_{z}\right)^{2}
$$

на дне погранслоя. Дело в том, что на дне погранслоя для вязких жидкостей должно быть выполнено условие прилипания. При условии прилипания (для несжимаемых жидкостей) [22] получено, что на гладком участке обтекаемой поверхности выполнено равенство

$$
\Omega^{2}-\left(\nabla V_{x}\right)^{2}-\left(\nabla V_{y}\right)^{2}-\left(\nabla V_{z}\right)^{2}=0 .
$$

Следовательно, на дне погранслоя правая часть (2.4) меньше нуля. В силу непрерывности она останется таковой нуля и в некотором слое вблизи дна. В настоящей работе этот слой будем называть слоем самогравитаџии или $G$-слоем.

Таким образом, вблизи гладкого участка поверхности, обтекаемого несжимаемой вязкой жидкостью, есть слой ненулевой толщинь (G-слой), в котором давление жидкости (если оно непостоянно) не может достигать минимального значения.

Полученный результат (существование $G$-слоя) и результат $Г$. Гамеля (течение с малой завихренностью) несопоставимы, поскольку на дне погранслоя величина завихренности, в общем случае, не мала.

Из существования обнаруженного в данной работе критерия $G$-слоя следует, что через этот слой не могут проходить минимумы в волнах и солитонах давления (в нестационарных процессах). Это справедливо для областей с гладкими параметрами. Например, вся зона отрыва такой областью не является. А части этой зоны, разделенные линиями разрыва, могут рассматриваться как зоны гладких параметров. Для них сформулированный выше принцип минимума давления в $G$-слое верен, и невозможно прохождение волн и солитонов с минимумами давления.

\section{7. Заключение}

В работе замечено (и в этом состоит новизна работы), что с учетом самогравитации во многих важных течениях можно априори указать область течения с отрицательным значением правой части (2.4). В такой области минимум давления достигается на границе и только на границе рассматриваемого течения. Этот вывод качественно отличается от многих других 
принципов минимума давления, в которых также утверждается, что минимум давления достигается на границе, но допускается случай постоянства давления во всей области.

Полученные результаты свидетельствуют, что учет самогравитации в некоторых случаях, может приводить к новым качественным выводам, основанным на исследовании знака правой части уравнения (2.4).

\section{Литература}

1. Ландау Л. Д., Лифшиц Е. М. Теоретическая физика. Т. 6. Гидродинамика. М. : ФИЗМАТЛИТ, 2001. - 736 с.

2. Privalova V. V., Prosviryakov E. Yu. Vortex flows of a viscous incompressible fluid at constant vertical velocity under perfect slip conditions // Diagnostics, Resource and Mechanics of materials and structures. - 2019. - Iss. 2. - P. 57-70. - DOI: 10.17804/2410-9908.2019.2.057-070. URL: https://dream-journal.org/DREAM_Issue_2_2019_Privalova_V.V._et_al._057_070.pdf

3. Prosviryakov E. Yu. Dynamic equilibria of a nonisothermal fluid // Vestn. Samar. Gos. Tekhn. Univ., Ser. Fiz.-Mat. Nauki [J. Samara State Tech. Univ., Ser. Phys. Math. Sci.]. - 2018. Vol. 22, no. 4. - P. 735-749. - DOI: 10.14498/vsgtu1651.

4. Просвиряков Е. Ю. Точные решения трехмерных потенциальных и завихренных течений Куэтта вязкой несжимаемой жидкости // Вестник Национального исследовательского ядерного университета МИФИ. - 2015. - Т. 4. Вып. 6. - С. 501-506. - DOI: 10.1134/S2304487X15060127.

5. Burmasheva N. V., Prosviryakov E. Y. Thermocapillary Convection of a Vertical Swirling Liquid // Theoretical Foundations of Chemical Engineering. - 2020. - Vol. 54. - P. 230-239. DOI: 10.1134/S0040579519060034.

6. Prosviryakov E. Y. New class of exact solutions of Navier-Stokes equations with exponen_tial dependence of velocity on two spatial coordinates // Theoretical Foundations of Chemical Engineering. - 2019. - Vol. 53, No. 1. - P. 107-114. - DOI: 10.1134/S0040579518060088

7. Aristov S. N., Knyazev D. V., Polyanin A. D. Exact solutions of the Navier-Stokes Equations with the linear dependence of velocity components on two space variables // Theoretical Foundations of Chemical Engineering. - 2009. - Vol. 43. No. 5. - P. 642-662. DOI: 10.1134/S0040579509050066.

8. Knyazev D. V. Solving the motion equations of a viscous fluid with a nonlinear dependence between a velocity vector and some spatial variables // Journal of Applied Mechanics and Technical Physics. - 2018. - Vol. 59. - P. 928-933. - DOI: 10.1134/S0021894418050218.

9. Hamel G. Ein allgemeiner Satz über den Druck bei der Bewegung volumbeständiger Flüssigkeiten // Monatsh. f. Mathematik und Physik. - 1936. - Vol. 43. - P. 345-363. DOI: $10.1007 / \mathrm{BF} 01707614$.

10. Бэтчелор Дж. Введение в динамику жидкости / пер. с англ. - М. : Мир, 1973. - 778 с.

11. Трусделл К. Первоначальный курс рациональной механики сплошных сред / пер. с англ. - М. : Мир, 1975. - 592 с.

12. Серрин Дж. Математические основы классической механики жидкости / пер. с англ. А. Б. Шабата ; под ред. Л. В. Овсянникова. - М. : Изд-во иностранной литературы, 1963. $256 \mathrm{c}$.

13. Седов Л. И. Механика сплошной среды. Т. 2. - М. : Наука, 1973. - 568 с.

14. Сизых Г. Б. Признак наличия точки торможения в плоском безвихревом течении идеального газа // Труды МФТИ. - 2015. - Т. 7, № 2. - С. 108-112.

15. Бурмистров А. Н., Ковалев В. П., Сизых Г. Б. Принцип максимума для решения уравнения эллиптического типа с неограниченными коэффициентами // Труды МФТИ. - 2014. Т. 6, № 4. - C. 97-102.

16. Golubkin V. N., Sizykh G. B. Maximum principle for the Bernoulli function // TsAGI Science Journal. - 2015. - Vol. 46, No. 5. - P. 485-490. - DOI: 10.1615/TsAGISciJ.v46.i5.50. 
17. Голубкин В. Н., Сизых Г. Б. Принципы максимума в гидродинамике // XI Всероссийский съезд по фундаментальным проблемам теоретической и прикладной механики, Казань, 20-24 августа 2015 г. : сборник трудов. - Казань : Изд-во Казанского (Приволжского) федерального университета, 2015. - С. 989-991.

18. Беспорточный А. И., Бурмистров А. Н., Сизых Г. Б. Вариант теоремы Хопфа // Труды МФТИ. - 2016. - Т. 8, № 1 - С. 115-122.

19. Golubkin V. N., Kovalev V. P., Sizykh G. B. Maximum principle for pressure in ideal incompressible fluid flows // TsAGI Science Journal. - 2016. - Vol. 47, iss. 6. - P. 599-609. DOI: $10.1615 /$ TsAGISciJ.2017019567.

20. Sizykh G. B. A velocity minimum in a potential fluid flow // Fluid Dynamics. - 2017. 52 (3). - P. 345-350. - DOI: 10.1134/S0015462817030024.

21. Поляченко В. Л., Фридман А. М. Равновесие и устойчивость гравитирующих систем. М. : Наука, 1976. - 447 с.

22. Golubkin V. N., Korolev G. L., Sizykh G. B. Differential properties of pressure in a viscous fluid // TsAGI Science Journal. - 2016. - Vol. 47, iss. 1. - P. 41-49. - DOI: 10.1615/TsAGISciJ.2016017060. 